\title{
Impact of depression and fatigue on relapsing remitting multiple sclerosis in Kingdom of Saudi Arabia
}

\author{
Foziah J. Alshamrani, MD, Mohammed F. Almuaigel, MBBS, Fahd A. Alkhamis, MD, Abdullah A. Alsulaiman, MD, \\ Noor M. AlMohish, MBBS, Abdullah F. Albuhassah, MBBS, Anwar S. AlZahrani, MBBS, Ashraf A. Mahmoud Zaher, MD.
}

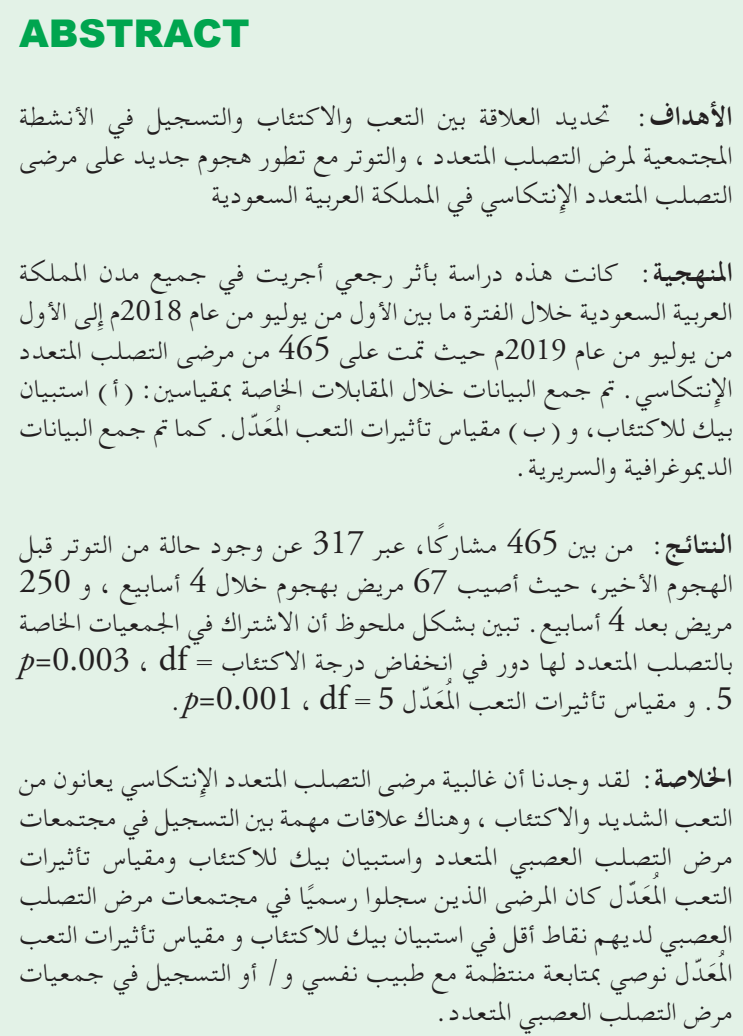

Objectives: To determine relationship between fatigue, depression with the registration in multiple sclerosis (MS) society activity, and stress with the risk developing a new attack in patients with Relapsing remitting MS (RRMS) in the Kingdom of Saudi Arabia (KSA).

Methods: This was a cohort retrospective study conducted in the KSA between July 2018 and July 2019 which included a total of 465 RRMS patients. Data were collected during interviews using the Beck Depression Inventory (BDI) and Modified Fatigue Impacts Scale (MFIS). Demographic and clinical data were also collected.
Results: Of 465 participants, 317 expressed psychological stress before the last attack, 67 of whom developed an attack within 4 weeks, and 250 of whom developed an attack after 4 weeks. Significantly lower BDI scores were associated with registration in MS associations $(p=0.003, \mathrm{df}=5)$. Significantly lower MFIS scores were associated with registration in MS associations $(p=0.001, \mathrm{df}=5)$.

Conclusion: The majority of RRMS patients have a significant fatigue and depression, and there are significant relationships between registration in the MS society and MFIS and BDI scores where patients who officially registered in MS society have lower score in MFIS and BDI. we recommend regular follow-ups with a psychologist and/or registration with MS societies.

Keywords: multiple sclerosis, relapsing remitting, fatigue, depression, Kingdom of Saudi Arabia, multiple sclerosis society

Saudi Med J 2020; Vol. 41 (3): 290-295 doi: 10.15537/smj.2020.3.24910

From the Department of Neurology (Alshamrani, Alkhamis, Alsulaiman), College of Medicine Imam Abdulrahman Bin Faisal University, King Fahad University Hospital; from the College of Medicine (Almuaigel, AlMohish), from the Department of Neurology (Zaher) College of Medicine, King Faisal University, Al Ahsa, from the Department of Orthopedic (Albuhassah), King Fahad Hospital, Al Hofuf; and from the College of Medicine (AlZahrani) Imam Abdulrahman Bin Faisal University, Dammam, Kingdom of Saudi Arabia.

Received 12th September 2019. Accepted 6th January 2020.

Address correspondence and reprint request to: Dr. Mohammed F. Almuaigel, College of Medicine, King Faisal University, Al Ahsa, Kingdom of Saudi Arabia. E-mail: al.muaigel@hotmail.com ORCID: https://orcid.org/0000-0001-6161-8179 
M ultiple sclerosis (MS) is considered as an autoimmune and demyelinating inflammatory disease that affects the central nervous system. ${ }^{1}$ It is considered as the main cause of disability in young people. $^{2}$ The disease is associated with various clinical manifestations that enable healthcare professionals to diagnose it accurately. ${ }^{3}$ Whilst the etiology of the disease remains unknown, multiple theories highlight genetic, environmental, and infectious factors that play a role. ${ }^{4}$

Multiple sclerosis is classified into 3 subtypes, the most common of which is relapsing remitting MS (RRMS). This disease is characterized by relapse periods followed by periods of complete recovery, which are usually accompanied by some residual deficit or sequel. ${ }^{5}$ Although relapses can cause serious residual disability, usually during the relapse period the disease usually progresses very little. It is the most common type of MS; it represents between $85-90 \%$ of cases. ${ }^{6}$

The major disabling symptoms of RRMS disease include mood disorder, ${ }^{7}$ cognitive impairment, ${ }^{8}$ and sexual dysfunction. ${ }^{9}$ This indicates that the disease affects both neurological and neuropsychiatric function. ${ }^{10}$ These symptoms have a substantial impact on the individual's overall life. For instance, daily activities, working life, social life, and family life are all affected. As a result, this leads to a reduction of quality of life (QOL) and emotional disturbance. ${ }^{11}$

Most research is consistent in recognizing that MS patients are affected by 2 primary factors, depression and fatigue. Both lead to a reduction in the subject's QOL scores. ${ }^{12,13}$ Studies on depression in RRMS patients show that approximately $2 / 3$ of patients with MS have or experience affective distancing to a greater or lesser degree. ${ }^{14}$ In the same way, patients with MS tend to be more prone to depression compared to other groups of patients suffering from some other chronic disease. ${ }^{15,16}$ Fatigue is defined as a state of physical and mental (cognitive) exhaustion, and that is not to provide the amount of activity that the individual performs. A study that conducted a survey of 223 Chinese patients showed that $86 \%$ of patients with MS have fatigue as symptoms. What allows to determine, that fatigue is the symptom that generates the difficulties or anxieties that they suffer. Which means that it is $65 \%$ higher than other symptoms. ${ }^{17}$

Disclosure. Authors have no conflict of interests, and the work was not supported or funded by any drug company.
Fatigue often interferes with the way the patient performs routine activities and is often associated with attacks. In addition, it is considered as a predecessor of the focal neurological features and may persist for a long period of time after the attack is over. ${ }^{18}$ It is unknown if there is any relation between the registration in MS society and the frequency of the attack, and the degree of fatigue and depression. The purpose of this study was to determine relationship between fatigue, depression with the registration in MS society activity, and stress with the risk developing a new attack in patients with RRMS in the Kingdom of Saudi Arabia (KSA).

Methods. This was a cohort retrospective study conducted across all cities of KSA between July 2018 and July 2019. It was ethically approved by Imam Abdulrahman Bin Faisal University, Dammam, Saudi Arabia. The sample size of 465 patients was determined based on prevalence and a confidence interval of $95 \%$. Patients were randomly selected. All patients' data were collected during interviews using the Beck Depression Inventory (BDI) ${ }^{19}$ and Modified Fatigue Impacts Scale (MFIS). ${ }^{20}$ Demographic and clinical data were subtracted from patients' hospital records. The study is according to the Declaration of Helsinki 2008.

The study included Saudi RRMS patients residing in the KSA. Inclusion criteria were as follows: patients with relapsing remitting multiple sclerosis (RRMS) who meet the diagnostic criteria of the 2010 revised McDonald criteria for the diagnosis of $\mathrm{MS} ;{ }^{21}$ aged $\geq 16$; patients should have a solid comprehensive reading ability and the ability to correlate content of scales, and able to answer questions.

Exclusion criteria were as follows: all patients who presented with any other chronic disease or comorbidity (this may have an impact on the scales); pregnancy or lactation; family history of depression, presenting with recent MS relapse in the previous 4 weeks, history of substance abuse, and presence of other neurological disorders resulted in patient exclusion from the study.

The demographic data that were considered during this study were age, gender, educational level, employment status, marital status, and course of disease.

The time of the last attack of the disease, registration with MS associations, and the psychological stress before the attack were assessed as clinical data.

Beck depression inventory. The BDI consists of 21 items. The items evaluate aspects such as mood, feeling of failure, pessimism, guilt, disgust, personal dissatisfaction, suicidal ideation, irritability, crying, selfaccusation, social isolation, perception of body image, labor difficulties, indecision, fatigue, insomnia, loss of 
appetite or weight, somatization of symptoms, worry and loss of sexual appetite. The description of the 21 items is categorized into 4 grades prioritized based on the severity or absence of the symptoms. The grades were assigned based on the values from 0-3; where: $0=$ no symptoms, $1=$ mild symptoms, $2=$ moderate symptoms, and, 3 = suffer from severe symptoms.

The degree of depression is categorized as follows: 0 to $10=$ normal ups and downs, $11-16=$ very slight alterations of mood, $17-20=$ there is a borderline depression, between 21-30 = moderate depression, $31-40=$ depression is severe, in a simple way, it can be said that the higher the result, the degree of depression is greater.

Modified fatigue impacts scale. The MFIS is the most appropriate method for measuring levels of fatigue in patients who have MS. This scale has 21 items, of which 9 items are somatic, 10 are cognitive and 2 items are mental. The elements are described based on 5 degrees of frequency, which go from absent to always. The values assigned for each item range from 0 to 4 : where $0=$ never, 1 = rarely, $2=$ sometimes, $3=$ often and $4=$ always or very frequently. It consists of 3 sub-scales: physical, cognitive, and psychosocial functioning. Recently, it has been recommended that a cutoff of 38 on the MFIS was most indicative of significant fatigue in MS. ${ }^{22}$ We considered Chi-square tests.

If there are any relationship between gender and MFIS and BDI scores, we used the Chi-square test for assessment.

Statistical analysis. The data were collected and analyzed using IBM Statistical Package for Social Sciences for Windows, version 19 (IBM Corp., Armonk, N.Y., USA). Descriptive analyses of the sociodemographic characteristics of the participants are presented in Table 1. Data were analyzed descriptively using simple statistical ratios consisting of frequency and percentages. A Pearson Chi-square was used. A $P$-value $<0.05$ was considered statistically significant.

Based on "Qualtrics", sample-size calculator, 368 patients is the minimal number of RRMS patients should be recruited in the general population of KSA (which was 8235 in 2016), and confidence intervals of $95 \%$ with a $5 \%$ margin of error.

Results. A total of 623 subjects were recruited in the study, with a response rate of $70 \%$. We excluded 158 subjects who met the exclusion criteria. Out of the remaining 465, 291 were females (62.6\%), and 174 were male (37.4\%). Most of the sample was concentrated in Central and Eastern districts (30.8\% for each), followed by Western $(22.2 \%)$, Norther $(8.8 \%)$, and Southern
Table 1 - Demographic data.

\begin{tabular}{lrr}
\hline Demographic data & \multicolumn{2}{c}{$\mathbf{n}(\%)$} \\
\hline Gender & 291 & $(62.6)$ \\
Female & 174 & $(37.4)$ \\
Male & 465 & $(100.0)$ \\
Total & & \\
& & \\
District & 143 & $(30.8)$ \\
Central district & 143 & $(30.8)$ \\
Eastern district & 41 & $(8.8)$ \\
Northern district & 32 & $(6.9)$ \\
Southern district & 3 & $(0.6)$ \\
Not limited to one district & 103 & $(22.2)$ \\
Western district & 465 & $(100.0)$ \\
Total & & \\
Multiple sclerosis (MS) society registration & 102 & \\
Officially registered with the MS Society & 363 & \\
Not registered with the MS Society & & \\
\hline
\end{tabular}

(6.9\%). A total of $0.6 \%$ were not confined to any one district. There were 102 subjects who were registered in the MS society (Table 1 ).

A modified version of the fatigue impact scale was used to determine the impact that fatigue has on the life of patients suffering from MS. The cutoff is 38 , if a patient has significant fatigue. Among 465 patients, 395 (84.94\%) had significant fatigue, where 70 (15.1\%) had less than 38 in the scoring system.

The BDI was used to assess depressive disorders in the sample of MS patients. The total score for the BDI is classified into scales. In the study, there were 110 patients classified as normal (23.7\%), 96 as borderline (20.6\%), 51 as mild (11\%), 129 as moderate (27.7\%), 46 as severe $(9.9 \%)$, and 33 as extremely severe $(7.1 \%)$.

Of the 317 patient who experienced psychological stress before the last attack, 67 developed an attack within less than 4 weeks, and 250 developed an attack within more than 4 weeks (Figure 1). A Pearson Chi-square test revealed a significant relationship between psychological stress and developing an attack $(p=0.002, \mathrm{df}=1)$, thereby showing that attacks are more likely among patients who face psychological stress.

A Pearson Chi-square test revealed a significant relationship between being registered in the MS society and MFIS scores $(p=0.001, \mathrm{df}=1)$, thereby showing that registration in the MS society is associated with lower MFIS score (Figure 2).

A Pearson Chi-square test revealed a significant relationship between registration in the MS society and MFIS scores $(p=0.003, \mathrm{df}=5)$, thereby showing 


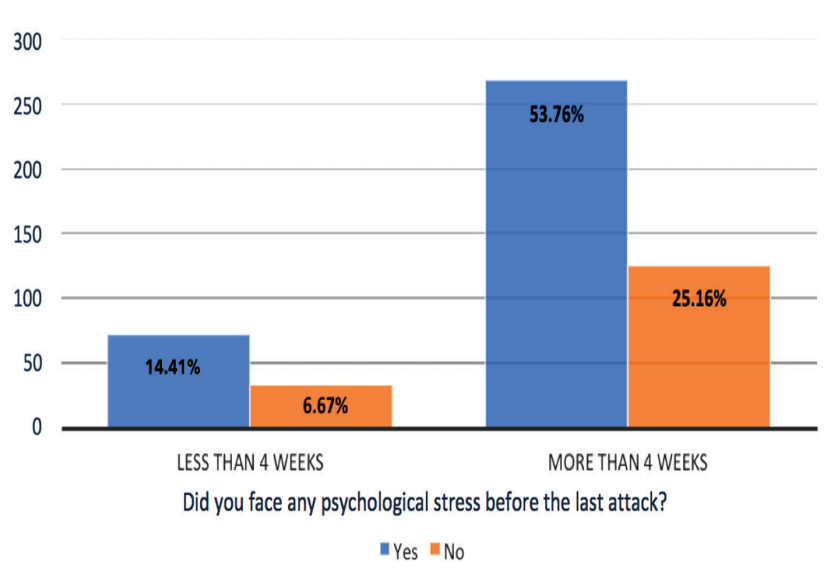

Figure 1 - Frequency of patients who experienced psychological stress before the last attack.

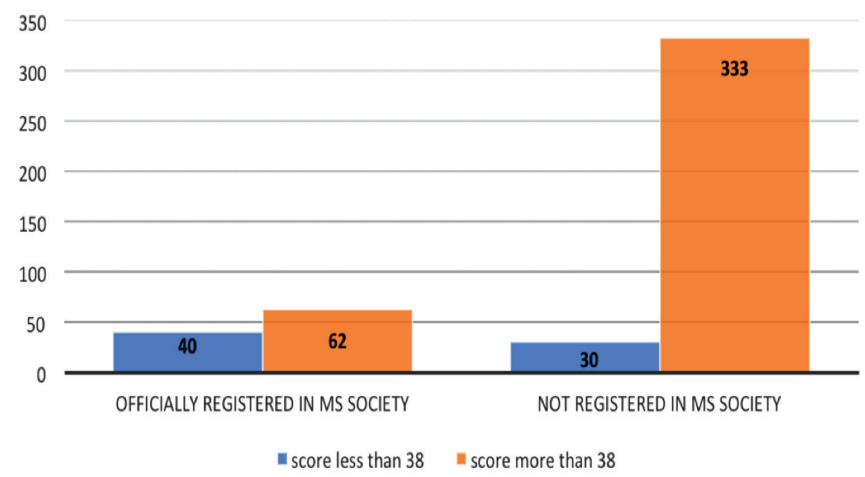

Figure $\mathbf{2}$ - The relationship between registration in the multiple sclerosis society and Modified Fatigue Impacts Scale scores.

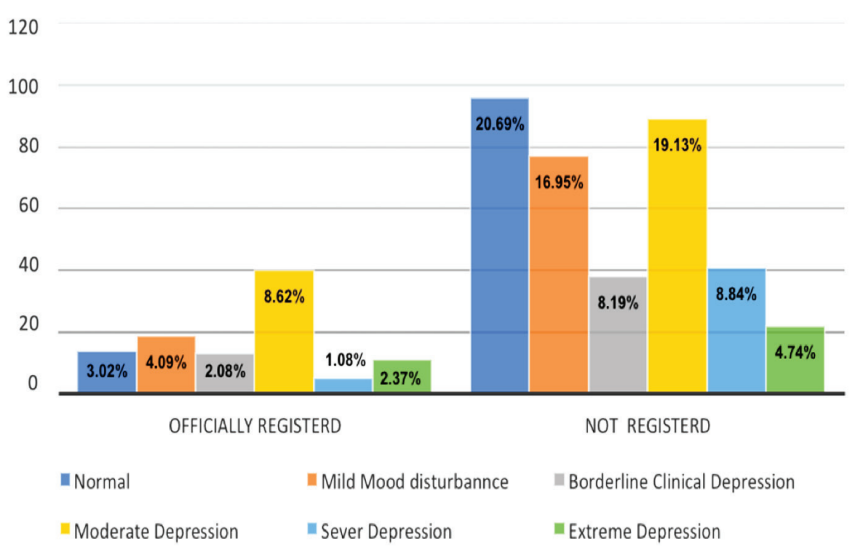

Figure 3 - The relationship between being registration in the multiple sclerosis society and Beck Depression Inventory scores. that registration in the MS society is associated with lower scores in the BDI (Figure 3).

A Pearson Chi-square test revealed no significant relationship between gender and MFIS scores $(p=0.55, \mathrm{df}=5)$. A Pearson Chi-square test revealed no significant relationship between gender and BDI scores $(p=0.26, \mathrm{df}=5)$.

Discussion. Many diseases and disorders are attributed to MS in addition to physical illness. Depression and fatigue are 2 major symptoms commonly associated with RRMS. The implications for future research and the objective of this study was to determine the relationship between fatigue, depression, and registration in the MS society activity (MSSA) in patients with RRMS in KSA, which very limited researches has been made on KSA. Our study has some limitations, it used subjective answers towards the questions instead of using objective tests, it only focused on one type of MS, and QOL score was not measured in the research, and the psychological stress must be measured as prospective rather than retrospective in order to state that psychological stress can increase the frequency of the attack.

Factors relevant to MS. In this study, the relationship between MFIS/BDI and gender were not statistically significant. However, we found statistically significant associations between MFIS/ $\mathrm{BDI}$ and MS society registration in which registration in the MS society is associated with lower scores in the MFIS/DI $(p<0.01)$. We also found a significant relationship between psychological stress and the risk of developing an attack. Several published papers have revealed a direct association between MS and mental disorders, ${ }^{7,9,11-13,17}$ These reports show that MS is not exclusively associated with MFIS and BDI scores but also other mental health disorders. For example, Razaz et $\mathrm{al}^{7}{ }^{7}$ showed that parental MS is significantly associated with the presence of anxiety disorders or mood in children of patients with MS. In Iran, they discovered that a significant factor with BDI-II scores was sexual dysfunction. ${ }^{?}$ An Australian study assessed mental and physical Health Related Quality of Life (HRQOL) in relation to "Prevalence of Comorbidities, Overweight and Obesity in an International Sample of People with Multiple Sclerosis and Associations with Modifiable Lifestyle Factors". They managed to demonstrate 
that many comorbidities are usually associated with the decrease in physical and mental HRQOL. On the other hand, overweight and obesity were associated with a lower HRQOL, controlling gender, age and degree of disability. ${ }^{11}$ On the other hand, Wijenberg et $\mathrm{a}^{12}$ discovered that patients who suffer from MS and have some degree of physical disability or depression tend to experience a higher level of fatigue. Solaro et $\mathrm{al}^{13}$ reported that the Expanded Disability Status Scale (EDSS) and the course that the disease follows, is a very important factor that determines BDI. Finally, Chen et $\mathrm{al}^{17}$ revealed that QOL is positively related to depression, disability and fatigue, therefore, the BDI rating greatly influences the QOL and mental health of patients with MS.

Prevalence of fatigue. According to our study which used the MFIS to assess the prevalence of fatigue among RRMS patients, we found that $85 \%$ of the patient had significant fatigue and $15.1 \%$ was below the cutoff score. While the incidence of severe fatigue in our study was moderately high, in the study by Wijenberg et al ${ }^{12}$ a percentage of $44 \%$ was obtained when calculating the total explained variance of fatigue by using the abbreviated fatigue questionnaire (AFQ). In contrast, Chen et $\mathrm{al}^{17}$ showed that $52 \%$ of MS patients show indications of fatigue.

Prevalence of depression. Depression is one of the precipitating factors of MS. Based on the BDI instrument, mild to moderate depression was detected in $38.7 \%$ of RRMS patients, whereas $17 \%$ of them had severe to extremely severe depression. This is consistent with a study published in Canada. ${ }^{7}$ They reported that $50 \%$ of the patients with MS experienced mental morbidity, where depression disorders were reported in $28.4 \%$ and anxiety in $17.6 \%$ of cases. Solaro et $\mathrm{a}^{13}$ reported higher rates of depression among MS patients. They reported that $46 \%$ of MS patients had minimal to moderate depression, whereas $45 \%$ had moderately severe to severe depression. Our results correspond with the study published by Čarnická et $\mathrm{al},{ }^{18}$ who reported a prevalence of $43 \%$ of MS patients with mild to moderate depression, and $12 \%$ with severe MS.

Measurement of depression. To measure the depression of RRMS patients, we used the BDI, developed by Beck et al. ${ }^{20}$ This consists of a 21-item questionnaire which covers different aspects related to depression. The 21 items were classified according to 4 grades, which reflect the severity or absence of the symptoms. Ratings are assigned a score from 0 to 3 . The level of depression is considered based on the following criteria: 1 to $10=$ normal, $11-16=$ very slight alterations of mood, 17-20 = borderline depression, and between 21-30 moderate depression, 31-40 = depression is severe, in a simple way, it can be said that the higher the result, the degree of depression is greater. Several published papers have used BDI to measure the degree of depression in patients with MS. ${ }^{9,13,17}$ However, Wijenberg et $\mathrm{al}^{12}$ applied the Hospital Anxiety and Depression Scale (HADS) to evaluate the level of depression and anxiety in MS patients.

Measurement of fatigue. We utilized the Modified Fatigue Impact Scale (MFIS) to quantify the level of fatigue in patients with RRMS, which was developed by Kos et al. ${ }^{21}$ This scale has 21 items, of which 9 items are somatic, 10 are cognitive, and 2 are mental. Responses are classified according to a 5-point rating scale which ranges from 0 (never) to 4 (often/always). Based on the criteria, the total MFIS score was classified as: $0-15=$ normal, $16-29=$ borderline, $30-43=$ mild, $44-57=$ moderate, $58-72=$ severe and 73-84 = extremely severe. Higher scores indicate more severe fatigue. The MFIS was used in a paper published by Chen et $\mathrm{al},{ }^{17}$ where it was also used to assess the severity of the fatigue among patients who suffers MS. However, this does not correspond to the study published by Wijenberg et al, ${ }^{12}$ where they applied the AFQ to assess the fatigue of MS patients. In contrast, in Slovakia, they used the Fatigue Severity Scale (FSS) to measure the fatigue of patients with MS. ${ }^{18}$

Multiple sclerosis societies. We found that the patient with RRMS is better to be registered in these kinds of societies in which significant lower MFIS and BDI scores were associated with registration in MS societies $(p=0.003, \mathrm{df}=5)$.

Study limitations. Our study used subjective answers towards the questions instead of using objective tests, it only focused in one type of MS, and QOL score was not measured in the study, and the psychological stress must be measured as prospective rather than retrospective in order to state that psychological stress can increase the frequency of the attack.

In conclusion, this study was conducted with the purpose of evaluating the existence of a relationship between fatigue, depression suffering from RRMS in Saudi Arabia. We screened male and female patients from different areas of Saudi Arabia. No significant relationship was found between gender and fatigue or depression. Whereas, a significant relationship was found between the stress and developing MS attacks. In addition, the study cooperated with MS societies to determine the impact of psychological support on the course of the disease. Registration in the MS 
society correlated with less MFIS/BDI scores. We recommend regular follow-ups with psychologists to manage depression, and fatigue as well as attendance at any social in order decreasing the level of fatigue and depression in patients with RRMS.

Acknowledgment. We would like to thank Arfa Ms Society, Mohammed Almulhim, Zainab Alahrani, Ibrahim Almajed, Abdulelah Alrashidi, Lama Almujalli, Munirah Alkrisi, Zahrah Aljardah for data collection and Narjes Alramadhan for statistical analysis. We would like also to acknowledge www. Editage.com for English language editing.

\section{References}

1. Mohebi N, Moghaddasi M, Zaribafian M. Relapsing remitting multiple sclerosis in an Iranian patient with neurofibromatosis type I. Neurol Int 2015; 7: 5966.

2. Barone DA, Singer BA, Merkov L, Rametta M, Suarez G. Survey of US patients with multiple sclerosis: Comparison of the new electronic interferon beta- $1 \mathrm{~b}$ autoinjector (BETACONNECTTM) with mechanical autoinjectors. Neurol Ther 2016; 5: 155-167.

3. Geisseler O, Nyffeler T, Linnebank M, Pflugshaupt T. Cognitive Impairment in Multiple Sclerosis: Clinical Manifestation, Neuroimaging Correlates, and Treatment. Semin Neurol 2016; 36: 203-211.

4. Morandi E, Tarlinton RE, Gran B. Multiple Sclerosis between Genetics and Infections: Human Endogenous Retroviruses in Monocytes and Macrophages. Front Immunol 2015; 6: 647.

5. Lublin FD. The incomplete nature of multiple sclerosis relapse resolution. J Neurol Sci 2007; 256: S14-S18.

6. Richards RG, Sampson FC, Beard SM, Tappenden P. A review of the natural history and epidemiology of multiple sclerosis: implications for resource allocation and health economic models. Health Technol Assess (Rockv) 2002; 6: 1-73.

7. Razaz N, Tremlett H, Boyce T, Guhn M, Marrie RA, Joseph KS. Incidence of mood or anxiety disorders in children of parents with multiple sclerosis. Paediatr Perinat Epidemiol. 2016; 30: 356-366.

8. Polychroniadou E, Bakirtzis C, Langdon D, Lagoudaki R, Kesidou E, Theotokis P, et al. Validation of the brief international cognitive assessment for multiple sclerosis (BICAMS) in Greek population with multiple sclerosis. Mult Scler Relat Disord 2016; 9: 68-72.

9. Mohammadi K, Rahnama P, Mohseni SM, Sahraian MA, Montazeri A. Determinants of sexual dysfunction in women with multiple sclerosis. BMC Neurol 2013; 13: 83.
10. Barak Y, Gabbay U, Glad R, Sarova-Pinhas I, Achiron A. Neuropsychiatric assessment as a secondary outcome measure in a multiple sclerosis intravenous immunoglobulin L(IMg) trial. Int J Psychiatry Clin Pract 1999; 3: 31-34.

11. Marck CH, Neate SL, Taylor KL, Weiland TJ, Jelinek GA. Prevalence of comorbidities, overweight and obesity in an international sample of people with multiple sclerosis and associations with modifiable lifestyle factors. PLoS One 2016; 11: e0148573.

12. Wijenberg MLM, Stapert SZ, Köhler S, Bol Y. Explaining fatigue in multiple sclerosis: cross-validation of a biopsychosocial model. J Behav Med 2016; 39: 815-822.

13. Solaro C, Trabucco E, Signori A, Martinelli V, Radaelli M, Centonze D, et al. Depressive symptoms correlate with disability and disease course in multiple sclerosis patients: an Italian multi-center study using the beck depression inventory. PLoS One 2016; 11: e0160261.

14. Ryan JJ, Umfleet LG, Gontkovsky ST. Prorating WAIS - IV summary scores for patients with relapsing-remitting multiple sclerosis. Int J Neurosci. 2015; 126: 1025-1029.

15. Mohr DC, Goodkin DE, Gatto N, Van Der Wende J. Depression, coping and level of neurological impairment in multiple sclerosis. Mult Scler J 1997; 3: 254-258.

16. Patten SB, Beck CA, Williams JVA, Barbui C, Metz LM. Major depression in multiple sclerosis: A population-based perspective. Neurology 2003; 61: 1524-1527.

17. Chen K, Fan Y, Hu R, Yang T, Li K. Impact of Depression, Fatigue and Disability on Quality of Life in Chinese Patients with Multiple Sclerosis. Stress Heal 2012; 29: 108-112.

18. Čarnická Z, Kollár B, Šiarnik P, Krížová L, Klobučníková K, Turčáni P. Sleep disorders in patients with multiple sclerosis. J Clin Sleep Med 2015; 11: 553-557.

19. Beck AT, Guth D, Steer RA, Ball R. Screening for major depression disorders in medical inpatients with the Beck Depression Inventory for Primary Care. Behav Res Ther 1997; 35: 785-791.

20. Kos D, Kerckhofs E, Carrea I, Verza R, Ramos M, Jansa J. Evaluation of the modified fatigue impact scale in four different European countries. Mult Scler J 2005; 11: 76-80.

21. Polman CH, Reingold SC, Banwell B, Clanet M, Cohen JA, Filippi M, et al. Diagnostic criteria for multiple sclerosis: 2010 Revisions to the McDonald criteria. Ann Neurol 2011; 69: 292-302.

22. Strober, Lauren B. "Fatigue in multiple sclerosis: a look at the role of poor sleep." Frontiers in neurology 2015; 6: 21. 